\title{
The Influence of the Stage of Differentiation of the Gut on the Migration of Neural Cells: An Experimental Study of Hirschsprung's Disease
}

\author{
J. H. CAREL MEIJERS, DICK TIBBOEL, ARTHUR W. M. VAN DER KAMP, \\ ILSE C. C. M. VAN HAPEREN-HEUTS, PAUL KLÜCK, AND JAN C. MOLENAAR \\ Department of Pediatric Surgery [J.H.C.M., D.T., I.C.C.M.vH., P.K., J.C.M.] and Department of Cell Biology \\ and Genetics [A.W.M.v.d.K.], Erasmus University School of Medicine, Rotterdam, The Netherlands
}

\begin{abstract}
Based on experimental studies in mutant mouse strains, an imbalance between the rate of migration of neural crest cells and the rate of differentiation of the mesenchyme of the distal gut has been proposed as an etiological factor in Hirschsprung's disease. We studied the influence of the stage of differentiation of embryonal chick gut on the migration of neural crest cells in an in vivo culture system: the chorioallantoic membrane. Neural crest cells in cultured gut were demonstrated with antibodies directed against the HNK-1 epitope. Enteric neurons were demonstrated with neurofilament immunoreactivity. By culturing isolated gut segments of $\mathrm{E} 4$ embryos, we obtained aneuronal (neurofilament-negative) embryonal chick gut up to 25 days of development. In cocultures of aneuronal gut and the neural anlage (neural tube and neural crest) neural crest cell colonization was observed, even in advanced stages of differentiation. The significance of the results is discussed in terms of the etiology of Hirschsprung's disease. (Pediatr Res 21: 466-470, 1987)
\end{abstract}

\section{Abbreviations}

E, embryonal day

Ex $+y$, embryonal day $x+y$ days culture

CAM, chorioallantoic membrane

HNK-1, human natural killer cell

NA, neural anlage

NF, neurofilament

NC, neural crest

PBS, phosphate-buffered saline

HE, hematoxylin and eosin

\section{ETIOLOGY OF HIRSCHSPRUNG'S DISEASE}

Nearly $100 \mathrm{yr}$ after the first clinical description of Hirschsprung's disease (1) there is still little understanding of the etiology and pathogenesis. The basic defect is attributed variously to (a) intrinsic abnormalities of migrating NC cells (2), (b) muscular dysplasia of mesenteric vessels $(3,4)$; or (c) antenatal viral destruction of enteric neurons (5). In an embryological study of the piebald lethal mouse strain with aganglionosis of the distal colon, resembling Hirschsprung's disease, an apparent slowing of the proximo-distal sequence of the nonspecific esterase stain-

Received July 14, 1986; accepted December 9, 1986.

Correspondence to Dr. D. Tibboel, Department of Pediatric Surgery, Sophia Children's Hospital, P.O. Box 70029, 3000 LL Rotterdam, The Netherlands.

Supported by Grant 85-52 from the Sophia Foundation for Medical Research. ing of NC cells was noted (6). It was surmised that aganglionosis had been caused by NC cell migration being out of phase with the longitudinal growth and differentiation of the developing gut. Studies with the lethal spotted mouse strain provided experimental evidence for the localization of the effect of the 1s gene in the distal gut (7). We investigated whether mere temporal factors in the distal gut hamper the NC cell colonization of the gut. An advanced stage of differentiation of the gut mesenchyme might inhibit the normal invasion of NC cells.

\section{NC CELL MIGRATION TO DEVELOPING GUT}

Little is known about NC cell migration in mammalian embryos, but it has been studied extensively in the chicken embryo (8-10). When the vagal NC is deleted, the distal gut becomes aganglionic (11). Studies with chick-quail chimeras demonstrated that most enteric neurons derive from the NC opposite somite 1-7 (8). These NC cells use a pathway between the ectoderm and the somite to reach the region of the third branchial arch. They enter the developing gut at the level of the pharynx (12).

At the axial level of early embryos, the normal process of $\mathrm{NC}$ cell migration can be affected by temporal factors $(13,14)$. We studied the influence of temporal factors on the migration of NC cells at the peripheral level, in the embryonal postumbilical gut.

\section{IN VIVO CULTURES OF EMBRYONAL GUT}

We used the nutritive properties of the CAM of the chicken embryo to culture segments of embryonal gut. In comparison with in vitro culture systems, CAM cultures have the advantage in that grafted embryonal tissues follow an almost normal course of differentiation (15). CAM vessels anastomose with the developing vessels in the graft, resulting in normal vascularization. The grafting technique is reviewed in detail by Coulombre (16).

The postumbilical gut of E4 embryos contains no NC cells (17). We obtained aneuronal gut of different stages of development by culturing postumbilical gut of $\mathrm{E} 4$ embryos for different periods of time. When an isolated segment of embryonal gut is cultured on the CAM in combination with the NA (NA = neural tube $+\mathrm{NC}$ ) of an E2 embryo, the segment is colonized and innervated in a physiological fashion (18).

Monoclonal antibodies have recently been introduced that stain (migrating) NC cells or enteric neurons selectively. Most NC cells express an epitope, which is recognized by antibodies produced by the HNK-1 hybridoma cell line (19). At the vagal level of the NC, a subpopulation of premigratory NC cells can be stained selectively with antibodies directed against NF epitopes $(20,21)$. We used HNK-1 epitopes for the identification of NC cells, while NF epitopes served as a marker for the neuronal subpopulation of NC cells in sections of cultured segments of embryonal gut. 


\section{MATERIALS AND METHODS}

Animals and stages of development. Eggs of white leghorn chickens (Gallus Domesticus) were incubated at $38^{\circ} \mathrm{C}$ in a forced draft incubator at a relative humidity of $80 \%$. For young embryos, the stage of development was determined by the number of paired somites, while for older embryos we used the table of Hamburger and Hamilton (22) or the number of days of incubation.

Removal of organs and tissues for transplantation. The hindgut (the region between the cecal primordia and the cloaca) was dissected from embryos before its colonization by NC cells, i.e. at day 4 and 5 of incubation. The ganglion of Remak, which is formed at stage 24-25 (23) and is located in the dorsal mesentery, was removed from the explants (E5 embryos) by dissection using tungsten needles.

Segments of the vagal neural tube (somite 1-7) together with the NC, were isolated from 12-21 somite embryos (E2) by using microdissection with tungsten needles after 10 min of trypinization (porcine trypsin $0.25 \%$ diluted in PBS at room temperature).

Transplantation techniques. Segments of aneuronal gut were grafted alone or in combination with vagal NA onto the CAM of 7-day-old chick embryos after abrasion of the superficial CAM layer, at the bifurcation of two great vessels. The grafts were fixed in position with a piece of cellophane (Clingo wrap sterilized in $70 \%$ ethanol and dried in air). The egg was sealed with Scotch tape and incubated for $1 \mathrm{wk}$.

Immunocytochemical techniques. Grafts were embedded in Tissue Tek II O.C.T. Compound (Miles) and snap-frozen in liquid nitrogen cooled isopentane. Frozen sections $(10 \mu \mathrm{m})$ were mounted on slides coated with chrome alum, fixated in acetone for $5 \mathrm{~min}$, and then dried in air. Other grafts were fixated in $4 \%$ formaldehyde in PBS, dehydrated, and embedded in paraffin. Sections $(7 \mu \mathrm{m})$ were deparaffinized, hydrated, and used for immunocytochemical investigation or stained with $\mathrm{HE}$.

Frozen sections were stained with three monoclonal antibodies raised against NF-triplet proteins. The 3G6 Ig-M antibody is specific for the $160 \mathrm{kD}$ and $200 \mathrm{kD}$ NF-protein (24), the $2 \mathrm{~F} 11$ antibody (Sanbio) for the $70 \mathrm{kD}(24)$ and C90 for the $200 \mathrm{kD}$. The antibody raised against the HNK-1 (Becton \& Dickinson) antigen was used on both paraffin and frozen sections. Rabbitantimouse peroxidase conjugated immunoglobulins (Dako) were used in a dilution of $1: 100$. In order to reduce background staining, we added $2 \%$ chick serum to the conjugate. Peroxidase was visualized by $0.1 \% 3,3^{\prime}$ diamino benzidine $4 \mathrm{HCl}$ (Serva) and $0.02 \%$ hydrogen peroxide. All rinsing and diluting was done in PBS (pH 7.4) with $0.1 \%$ Tween 20.

\section{RESULTS}

Localization of HNK-1 and NF epitopes in embryonal gut. Postumbilical gut of E4 embryos does not show HNK-1 or NF immunoreactivity. The expression of the HNK-1 antigen was first detected in the postumbilical gut of E6 embryos. No differentiated cell types were recognized at this stage in the mesenchyme of the gut. The stratified mucosal epithelium is surrounded by a mesenchyme that shows no regional specializations. The mesenchyme itself is enclosed within a simple cuboidal serosal epithelium. No nervous tissue was recognized histologically with $\mathrm{HE}$ staining.

3G6 immunoreactivity was first detected in postumbilical gut of E6 embryos. In the postumbilical segment of the gut of E7 embryos, the neurons of the myenteric and submucous plexus were identified with $3 \mathrm{G} 6$ anti-NF antibodies. Thus both the HNK-1 and the 3G6 anti-NF antibodies enabled detection of neuronal (precursor) cells at very early stages of development.

Staining of the C90 anti-NF antibody first appeared in postumbilical gut of E8 embryos, while the 2F11 anti-NF antibody only revealed nervous tissue in embryonal gut of advanced stages of differentiation, i.e. in E18 embryos.
Culture of differentiated embryonal aneuronal gut. We grafted postumbilical segments of embryonal gut of E4 embryos onto the CAM of E7 embryos. After 1 wk CAM culture, the segment showed a stratified epithelium. The mesenchyme had differentiated into a circular, smooth muscle layer. The lumen of the gut was circular. No NF or HNK-1 immunoreactivity was revealed, demonstrating the absence of enteric neurons and NC cells. Similarly, the postcecal part of E5 embryos did not show immunoreactivity with HNK-1 or anti-NF antibodies after 7 days culture (see Fig. 1). In the gut segment between the umbilicus and the ceca of E5 embryos, however, NC cells were visualized with HNK-1 immunoreactivity, while enteric neurons stained with the 3G6 NF-antibody (see Figs. 2, 3, and 7). In a series of experiments in which subsequent levels of the postumbilical gut were cultured on the CAM, we did not observe any staining in the distal parts of the gut prior to staining in the proximal parts.

In order to obtain aneuronal gut of advanced stages of development, the CAM-cultured aneuronal gut was freed from the surrounding membranes and grafted anew to the CAM of an E7 embryo. By subsequent subculturing, we obtained aneuronal gut of 11 and 18 and 25 days of development (see Fig. 6). In comparison with normal in situ development, the histological picture of these cultured gut segments corresponded to age. In cultured segments of E4 +14 , the lumen had lost its circular appearance. The stratification of the epithelium had changed

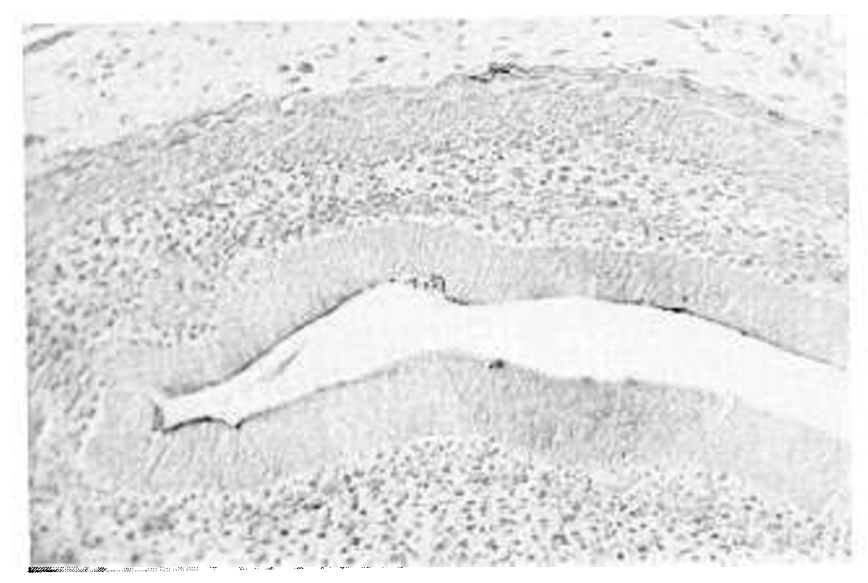

Fig. 1. Paraffin section of CAM-cultured postcecal gut of an E5 embryo. The circular smooth muscle layer and the epithelium have differentiated. There is no HNK-1 immunoreactivity, indicating the absence of NC cells. Magnification $\times 25$. Comparable age E12.

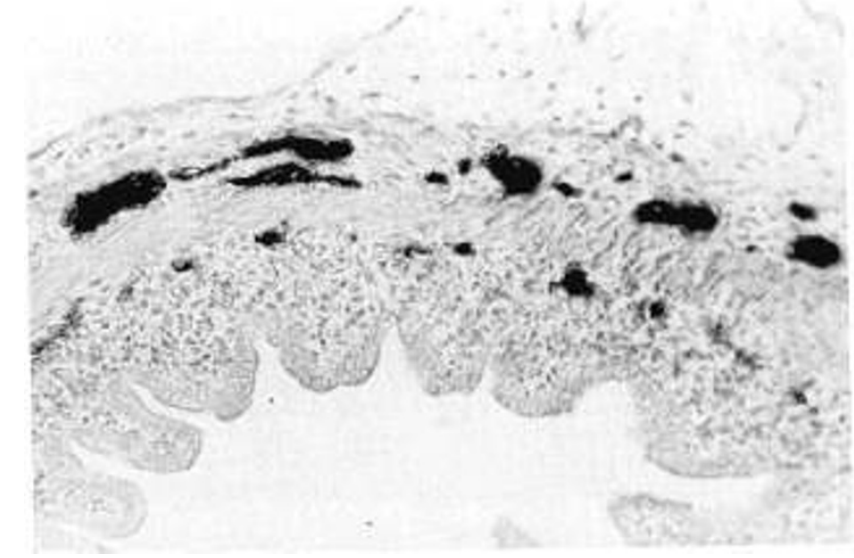

Fig. 2. Paraffin section of CAM-cultured prececal gut of an E5 embryo. Note the presence of HNK-1 immunoreactivity at the sites of the myenteric and submucous plexus. Magnification $\times 25$. Comparable age E12. 


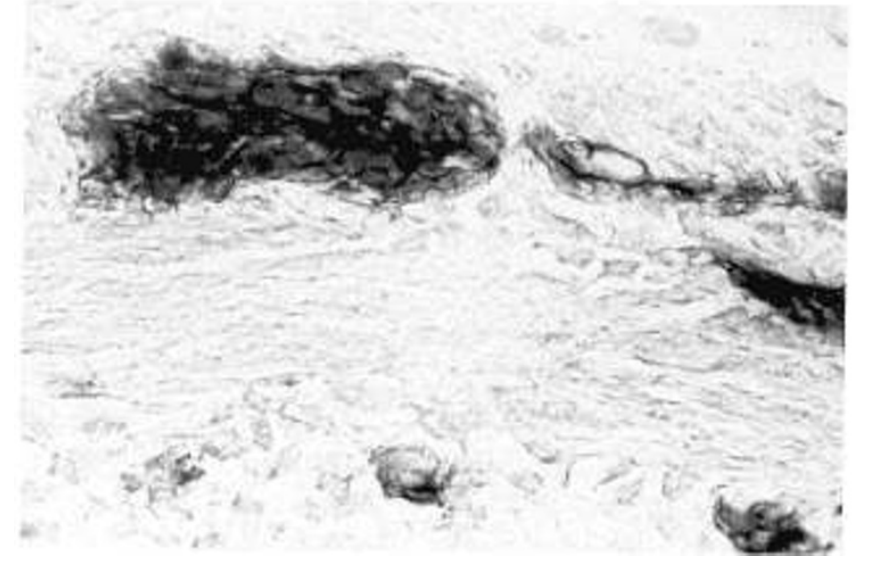

Fig. 3. Detail of the myenteric and submucous plexus of Figure 2 showing HNK-1 positive ganglioncells. Magnification $\times 100$.

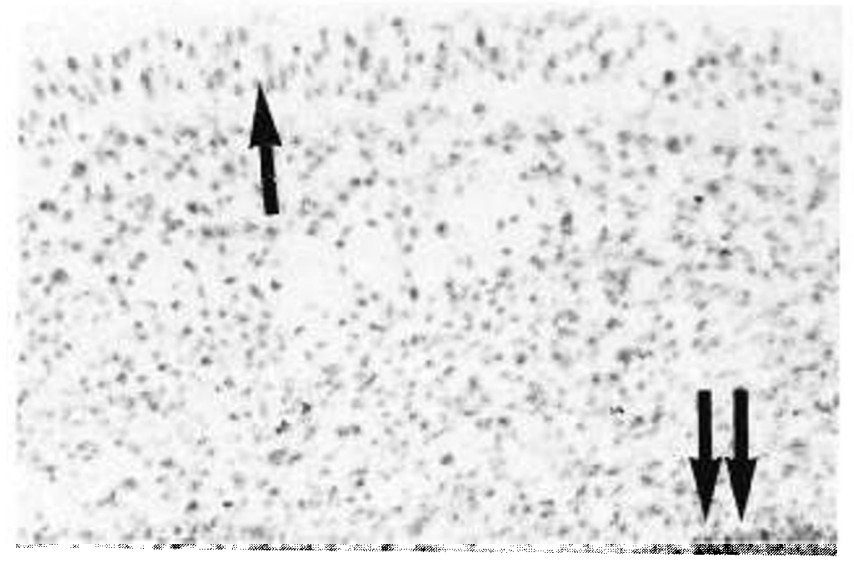

Fig. 4. Frozen section of postumbilical gut of an E4 embryo stained with $\mathrm{C} 90$ anti-NF antibody. No NF immunoreactivity is observed. The mesenchyme has no regional specializations. Arrow indicates the lumen. The double arrow indicates the serosal side. Magnification $\times 40$.

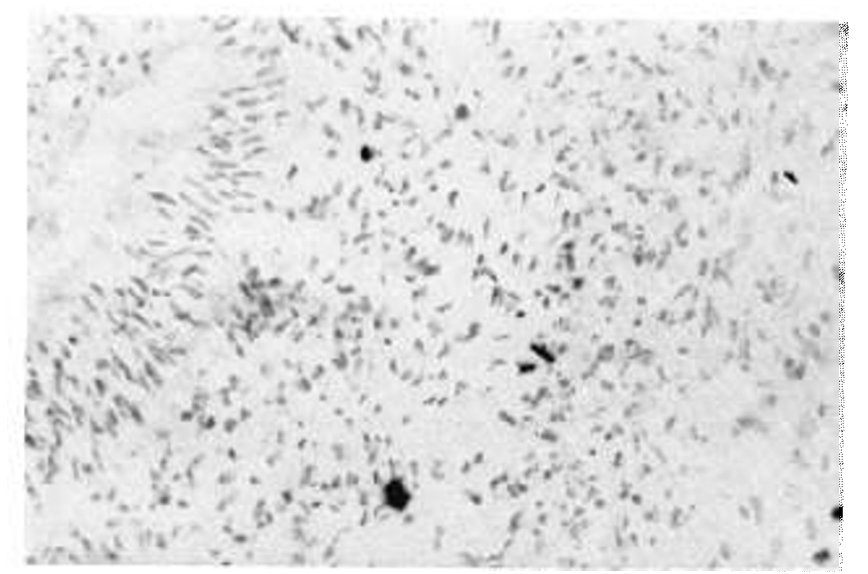

Fig. 5. Frozen section of postumbilical gut of an E4 embryo (cultured for 7 days on CAM) stained with anti-NF antibody C90. There is no NF immunoreactivity, indicating the absence of enteric neurons. Magnification $\times 40$. Comparable age E11. Compare with the paraffin section of the same segment of the gut (Fig. 1).

into a single layered cuboidal epithelium. The mesenchyme had differentiated into longitudinal and smooth muscle layers. Apparently, the grafting procedure had only minor effects on the normal course of differentiation (see Fig. 6).
The longitudinal growth of the graft decreased with the repetition of grafting. At removal at E4, the postumbilical segment of gut measured $1 \mathrm{~mm}$ in length. After 7 days CAM culture, the $1-\mathrm{mm}$ aneuronal gut had elongated to $3-4 \mathrm{~mm}$. For the second transplantation, this segment was divided into parts of $1 \mathrm{~mm}$ each, which were subsequently grafted and cultured. After 7 days culture, these segments had elongated to $2-3 \mathrm{~mm}$. These grafts were isolated and again divided into $1-\mathrm{mm}$ segments and grafted to the CAM. At the time of removal, after 21 days of culturing, these grafts measured approximately $1.5 \mathrm{~mm}$.

Coculture of vagal neural anlage and "old" aneuronal gut. To determine whether cultured aneuronal gut of advanced stages of differentiation would still accept NC cells, we cocultured the aneuronal gut with the vagal NA. Sections of the CAM of E7 and E16 did not stain with anti-NF antibodies. NF immunoreactivity did not appear in sections of postumbilical gut of $\mathrm{E} 4$ (see Fig. 4) and the cultures of E4 gut (see Fig. 5). NF immunoreactivity only appeared in cocultures of aneuronal gut of E4 with NA (see Figs. 7 to 9). Aneuronal gut of E4 + 7, E5 + 7, E4 +14 , and E5 +14 was also invaded by NC cells (see Table 1 and Figs. 7 to 9). The observed NF immunoreactivity was localized at the sites of the myenteric and submucous plexus. With relevance to the size of the ganglia, no major divergence was observed comparing cocultures of NA with aneuronal gut of $\mathrm{E} 4$ and $\mathrm{E} 4+7$. Cocultures of NA with aneuronal gut of E4+

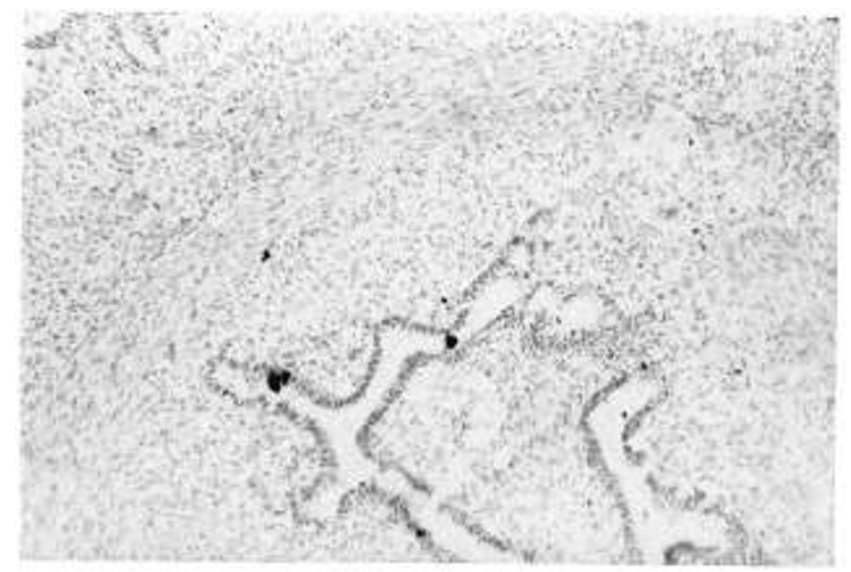

Fig. 6. Frozen section of aneuronal postumbilical gut of an E4 embryo, cultured for 21 days on CAM, stained with C90 anti-NF antibody. There is no NF immunoreactivity. Note the single layered cuboidal epithelium and the differentiated smooth muscle layer. Magnification $\times 16$. Comparable age 3 days after hatching.

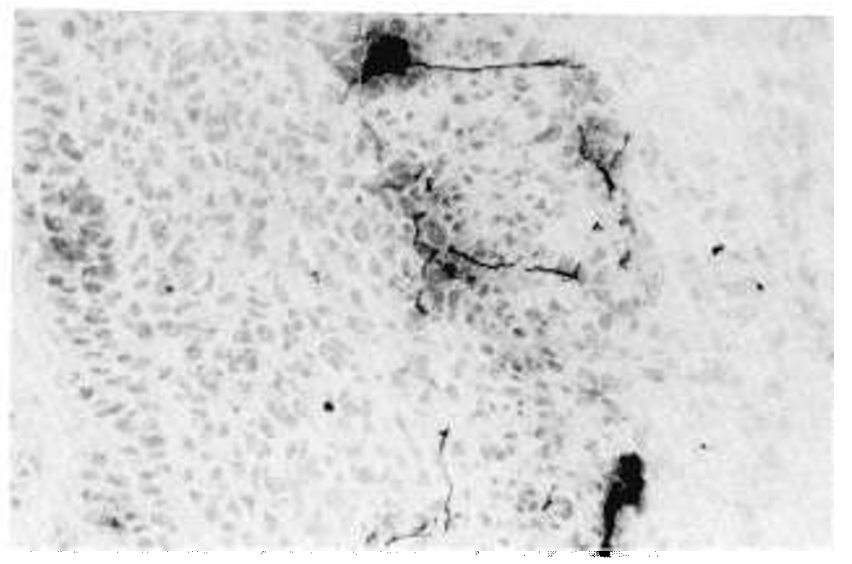

Fig. 7. Frozen section of a coculture (7 days) of postumbilical gut (E4 embryo) and NA (E2 embryo) stained with C90 anti-NF antibody. Note the NF immunoreactivity at the sites of the myenteric and submucous plexus. Magnification $\times 40$. Compare with Figure 5 . 


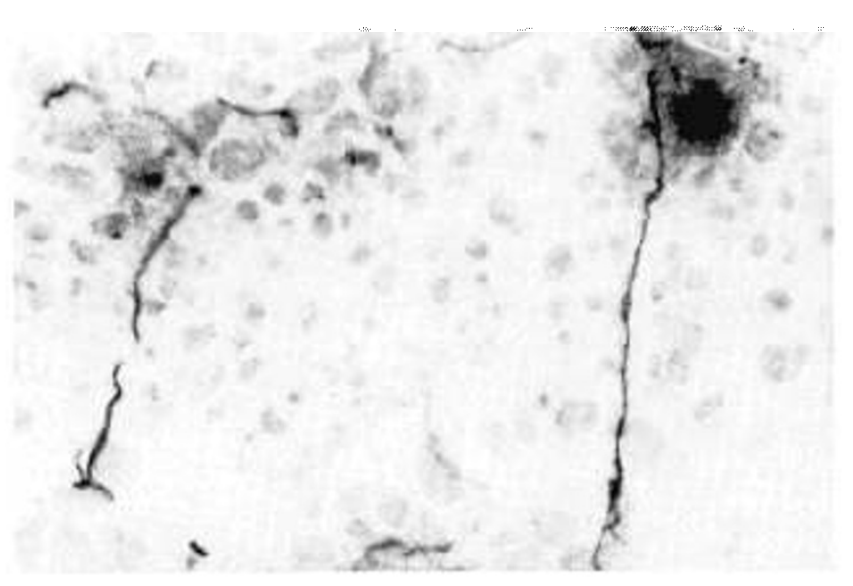

Fig. 8. Detail of Figure 7 showing NF immunoreactivity in cells and neurites in the submucous plexus. Magnification $\times 100$.

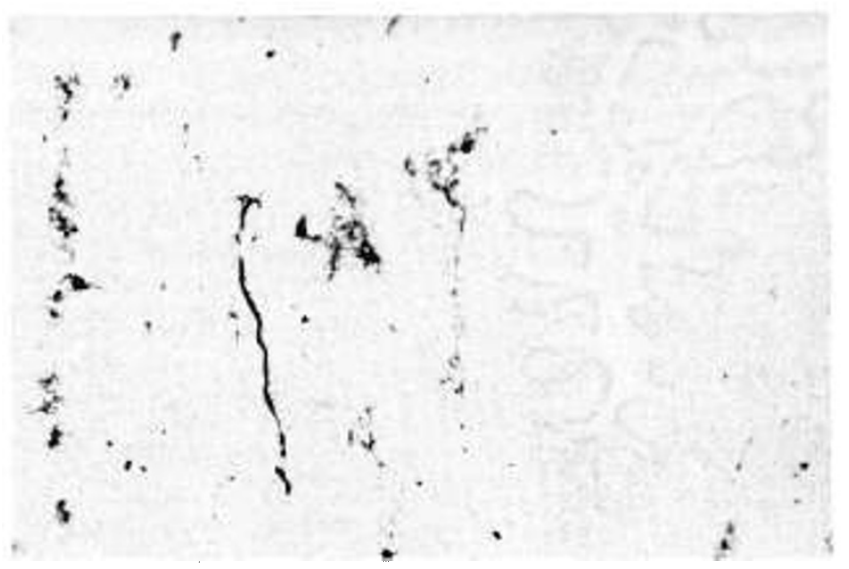

Fig. 9. Frozen section of a coculture (7 days) of aneuronal gut of 18 developmental days $(\mathrm{E} 4+14)$ and the NA (E2 embryo). Note C90 NF immunoreactivity at the sites of the myenteric and submucous ganglia. Magnification $\times 16$. Comparable age 3 days after hatching. Compare with Figure 6.

Table 1. Number of aneuronal bowel explants showing neurofilament positive nervous tissue after a 7 days coculture with neural anlage E2 on CAM

\begin{tabular}{ccccc}
\hline & \multicolumn{5}{c}{ Day of development (aneuronal gut) } \\
$\begin{array}{c}\text { Neurofilament } \\
\text { staining }\end{array}$ & $\begin{array}{c}4 \\
(n=8)\end{array}$ & $\begin{array}{c}5 \\
(n=8)\end{array}$ & $(n=12)$ & $(n=10)$ \\
\hline 3G6 & 7 & 7 & 10 & 8 \\
C90 & 7 & 7 & 9 & 8
\end{tabular}

Controls (cultures without neural anlage)

\begin{tabular}{ccccc} 
& $(n=13)$ & $(n=4)$ & $(n=3)$ & $(n=26)$ \\
\hline 3G6 & 1 & 0 & 0 & 3 \\
C90 & 1 & 0 & 0 & 3 \\
\hline
\end{tabular}

$* 11=4$ days in situ +7 days CAM culture.

$\uparrow 18=4$ days in situ +14 days CAM culture.

14 showed less NF immunoreactivity. Compared to sections of explanted gut of corresponding developmental stages the ganglia appeared to be smaller in all the cocultures.

\section{DISCUSSION}

NC cell invasion of "old" target organ. In the present study, we cultured aneuronal gut up to the age of $\mathrm{E} 4+21$ days. We observed NC cell invasion in both aneuronal gut of E4 and E4
+14 in cocultures with NA. In other words, NC invasion occurred despite a developmental difference of 14 days at the peripheral target organ. It is interesting to note that temporal factors do not hamper NC migration in a peripheral organ while they do at the axial level. Weston and Butler (12) transplanted radioactively labeled, neural tubes into progressively older recipients at the axial level. They found that migration of the implanted, labeled cells was restricted when the recipients were 9$12 \mathrm{~h}$ older with the $\mathrm{NC}$ cell migration already in progress. However, their conclusion was undermined by the damage inflicted during grafting, particularly in the case of older embryos. Bronner-Fraser \& Cohen (14) circumvented this problem by injecting cultured NC cells directly into somites. They (14) also observed that the migration was limited depending on the developmental age. The difference between the axial and peripheral levels can be explained by the assumption that at the axial levels the fibronectin- and laminin-rich migratory pathways are destroyed in a period of $9-12 \mathrm{~h}$ and that the migratory pathways are preserved in the postumbilical segment of the gut. It might also well be that in the distal gut, NC cells use other glycoproteins as substrates for migration than at the axial levels (13).

In cocultures of NA and aneuronal gut at different stages of development, the myenteric and submucous ganglia are of a comparable size. The ganglia in the cocultures, however, are smaller than those in the gut of corresponding ages. We presume that this is due to a loss of NC cells on the CAM or to the absence of factors stimulating $\mathrm{NC}$ cell proliferation in the CAM-cultured isolated segments of embryonal gut.

In the coculture experiments, we combined aneuronal gut and NA, two tissues that normally do not confront one another. It remains to be studied whether NC cells from the region of the third branchial arch and innervated gut will also colonize "old" aneuronal gut in CAM cultures. The NA of E2 embryos might contain a subpopulation of NC cells capable of migration on diverse migratory substrates. NC cells from older NC cell suppliers might be more selective as to the migratory substrate.

Etiological factors in Hirschsprung's disease. It has been demonstrated that, at least at the axial level, there are no major differences between the migratory pathways in chicken or mouse embryos, related to either the fibronectin or laminin lining (25). Furthermore, it is probable that NC cell migration in chicken embryos occurs in the same fashion as in mouse embryos, despite the fact that they use slightly different cell-adhesion molecules. For example, the HNK-1 epitope is not found on NC cells in mice (19). Therefore, we believe the results of our experiments with chicken embryos apply to the hypothesis of the development of aganglionosis in mice (6). As comparative research of the migratory pathways in man has not been reported to date, the relevance of our results to NC cell migration and the etiology of Hirschsprung's disease in man is entirely hypothetical.

The inaccessibility of differentiated distal parts of the gut for slowly migrating NC cells has been proposed as a causative factor of aganglionosis in the piebald lethal mouse (6) and for the aganglionosis in Hirschsprung's disease (26). The results of our experiments, indicate that the gut, at advanced stages of development, can still be colonized by NC cells. Consequently, it is not feasible that the migration of $\mathrm{NC}$ cells being out of phase with the longitudinal growth and differentiation of the gut is an etiological factor in Hirschsprung's disease in mice or in man.

Other factors such as intrinsic abnormalities of NC cells and structural defects within the embryonal gut remain possible. Further research in those directions must elucidate which of these etiologies has validity for Hirschsprung's disease.

Acknowledgments. The authors thank Alice Goslinga Ribbink, translator/stylistic editor, for her assistance in preparing the manuscript, Tar van Os for the microphotographs, and Dr. Sven O. Warnaar, Department of Pathology, State University Leiden, The Netherlands, for supplying the antineurofilament antibodies. 


\section{REFERENCES}

1. Hirschsprung $H 1887$ Stuhlträgheit Neugeborener infolge von Dilatation und Hypertrophie des Colons. Jahrbuch Kinderheilkunde 27:1-7

2. Bolande RP 1974 The neurocristopathies: a unifying concept of disease arising in neural crest maldevelopment. Hum Pathol 5:409-429

3. Taguchi T, Tanaka K, Ikeda K 1985 Fibromuscular dysplasia of arteries in Hirschsprung's disease. Gastroenterology 88:1099-1 103

4. Earlam R 1985 A vascular cause for Hirschsprung's disease? Gastroenterology 88:1274-1279

5. Hershlag A, Ariel I. Nissan S, Rosenmann E 1984 Cytomegalic inclusion virus and Hirschsprung's disease. $Z$ Kinderchir 39:252-254

6. Webster W 1973 Embryogenesis of the enteric ganglia in normal mice and in mice that develop congenital aganglionic megacolon. J Embryol Exp Morphol 30:573-585

7. Rothman TP, Gershon MD 1984 Regionally defective colonization of the terminal bowel by the precursors of enteric neurons in lethal spotted mutant mice. Neuroscience 12:1293-1311

8. LeDouarin NM 1982 The Neural Crest. Cambridge University Press, Cambridge

9. Duband JL, Thiery J-P 1982 Distribution in the early phase of avian cephalic neural crest migration. Dev Biol 93:308-323

10. LeDouarin NM 1984 Cell migrations in embryos. Cell 38:353-360

11. Yntema CL, Hammond WS 1954 The origin of intrinsic ganglia of trunk viscera from vagal neural crest in the chick embryo. J Comp Neurol 101:515541

12. Weston JA, Butler SL 1966 Temporal factors affecting localization of neural crest cells in the chicken embryo. Dev Biol 14:246-266

13. Tucker GC, Ciment G, Thiery JP 1986 Pathways of avian neural crest cell migration in the developing gut. Dev Biol 116:439-450

14. Bronner-Fraser M, Cohen AM 1980 Analysis of the neural crest ventral pathway using injected tracer cells. Dev Biol 77:130-141
15. Murray PDF 1928 Chorio-allantoic grafts of fragments of the two-day chick, with special reference to the development of the limbs, intestine and skin. Aust J Exp Biol Med Sci 5:237-256

16. Coulombre AJ 1967 Grafting of embryonic rudiments. In: Wilt FH, Wessels NK (eds) Methods in Developmental Biology. TY Crowell Co. New York, pp 457-469

17. Allan IJ, Newgreen DF 1980 The origin and differentiation of enteric neurons of the intestine of the fowl embryo. Am J Anat 157:137-154

18. Smith J, Cochard P, LeDouarin NM 1977 Development of choline acetyltransferase and cholinesterase activities in enteric ganglia derived from presumptive adrenergic and cholinergic levels of the neural crest. Cell Diff 6:199-216

19. Tucker GC, Aoyama H, Lipinski M, Tursz T, Thiery JP 1984 Identical reactivity of monoclonal antibodies $\mathrm{HNK}-1$ and $\mathrm{NCl}$ :conservation in vertebrates on cells derived from the neural primordium and on some leukocytes. Cell Diff 14:223-230

20. Tapscott SJ, Bennett GS, Holtzer H 1981 Neuronal precursor cells in the chick neural tube express neurofilament proteins. Nature 292:836-838

21. Payette RF, Bennett GS, Gershon MD 1984 Neurofilament expression in vagal neural crest derived precursors of enteric neurons. Dev Biol 105:272-287

22. Hamburger V, Hamilton HL 1951 A series of normal stages in the development of the chick embryo. J Morphol 188:49-92

23. Teillet MA 1978 Evolution of the lumbo-sacral neural crest in the avian embryo: origin and differentiation of the ganglionated nerve of Remak studied in interspecific quail-chick chimerae. W Roux Arch 184:251-268

24. Klück P, van Muijen GNP, van der Kamp AWM, Tibboel D, van Hoorn WA, Warnaar SO, Molenaar JC 1984 Hirschsprung's disease studied with monoclonal antineurofilament antibodies on tissue sections. Lancet 1:652-655

25. Sternberg J, Kimber SJ 1986 Distribution of fibronectin, laminin and entactin in the environment of migrating neural crest cells in early mouse embryos. J Embryol Exp Morphol 91:267-282

26. Okamoto E, Ueda T 1967 Embryogenesis of intramural ganglia of the gut and its relation to Hirschsprung's disease. J Pediatr Surg 2: 437-443 